\title{
Obesity in Mexico: prevalence, comorbidities, associations with patient outcomes, and treatment experiences
}

This article was published in the following Dove Press journal: Diabetes, Metabolic Syndrome and Obesity:Targets and Therapy

\author{
Marco D DiBonaventura' \\ Henrik Meincke ${ }^{2}$ \\ Agathe Le Lay ${ }^{2}$ \\ Janine Fournier ${ }^{2}$ \\ Erik Bakker ${ }^{3}$ \\ Allison Ehrenreich' \\ 'Kantar Health, New York, NY, \\ USA; ${ }^{2}$ Novo Nordisk, Copenhagen, \\ Denmark; ${ }^{3}$ Novo Nordisk, \\ Mexico City, Mexico
}

Correspondence: Henrik Meincke Novo Nordisk A/S, Vandtårnsvej I I4DK2860 Søborg Denmark

Tel: +4530798654

Email hhjh@novonordisk.com
Objective: The goal of this study is to investigate obesity and its concomitant effects including the prevalence of comorbidities, its association with patient-reported outcomes and costs, and weight loss strategies in a sample of Mexican adults.

Methods: Mexican adults $(\mathrm{N}=2,511)$ were recruited from a combination of Internet panels and street intercepts using a random-stratified sampling framework, with strata defined by age and sex, so that they represent the population. Participants responded to a survey consisting of a range of topics including sociodemographics, health history, health-related quality of life (HRQoL), work productivity, health care resource use, and weight loss.

Results: The sample consisted of 50.6\% male with a mean age of 40.7 years ( $\mathrm{SD}=14.5) ; 38.3 \%$ were overweight, and $24.4 \%$ were obese. Increasing body mass index (BMI) was associated with increased rates of type 2 diabetes, prediabetes, and hypertension, poorer HRQoL, and decreased work productivity. Of the total number of respondents, $62.2 \%$ reported taking steps to lose weight with $27.6 \%$ and $17.1 \%$ having used an over-the-counter/herbal product and a prescription medication, respectively. Treatment discontinuation rates were high.

Conclusion: Findings indicated that $62 \%$ of participants reported, at least, being overweight and that they were experiencing the deleterious effects associated with higher BMI despite the desire to lose weight. Given the rates of obesity, and its impact on humanistic and societal outcomes, improved education, prevention, and management could provide significant benefits. Keywords: obesity, quality of life, costs, treatment patterns, weight loss

\section{Introduction}

The prevalence of obesity in Mexico has risen substantially since the 1980s, with obesity now affecting over $30 \%$ of the adult population. ${ }^{1,2}$ It is projected that by 2050 , the proportion of obese men and women in Mexico will rise to $54 \%$ and $37 \%$, respectively, and more people will be obese than overweight. ${ }^{1}$ The rise in the rates of obesity has primarily been linked to an increased consumption of calorically dense foods and a more sedentary lifestyle. ${ }^{3-5}$

The presence of obesity is associated with a range of comorbidities including cardiovascular diseases, type 2 diabetes mellitus (T2DM), osteoarthritis, certain types of cancer, obstructive sleep apnea, and other conditions, ${ }^{1,2,6-11}$ which have a profound impact on the Mexican health care system. In 2010, costs were estimated to be $\$ 806$ million, and based on the projected increases in obesity rates, they could rise to $\$ 1.7$ billion by $2050 .^{1}$

Excess weight has also been associated with a number of impairments across psychosocial, symptom, and work-related domains. Research has suggested an 
association between increasing BMI and greater pain, ${ }^{12-15}$ as well as joint-related disorders such as osteoarthritis. ${ }^{16-18}$ Obesity has also been associated with higher rates of fatigue and sleep disorders. ${ }^{19-23}$ In the psychosocial domain, obese individuals have been reported to be at an increased risk of certain psychiatric illnesses, most notably depression, ${ }^{24}$ while overweight and obese individuals have been found to possess lower health-related quality of life (HRQoL) compared to those with a healthy weight. ${ }^{25}$ Finally, international research has consistently found an association between obesity and impairments in work productivity measures, with increasing BMI linked to greater absenteeism and presenteeism. ${ }^{7,26-29}$

Given the potential projected increases in obesity, it is also important to understand the weight loss actions taken by those who are overweight and obese in Mexico. Treatment of obesity is centered on optimizing food choices and improving exercise habits through lifestyle changes, for which there are numerous policy interventions. . $^{3,530,31}$ Weight loss interventions tend to follow a stepwise approach, with diet and lifestyle counseling as a foundation, before progressing to pharmacological and surgical approaches. ${ }^{32}$ There has been some suggestive evidence that involving family members in weight loss efforts could be beneficial, particularly within family-centered cultures. ${ }^{33}$ Despite global efforts to address obesity, sustainable weight loss remains elusive, and the majority of individuals will regain some or all of their lost weight over the course of 2 years. ${ }^{34}$ A survey study of Mexican university students reported that nearly $40 \%$ of respondents were attempting to lose weight; ${ }^{35}$ however, there exists little large-scale evidence of how frequently individuals with obesity in Mexico take steps to lose weight, the methods they use, or their satisfaction with these methods.

The goal of this study is threefold: 1) to explicate the prevalence of obesity, T2DM, prediabetes, and hypertension in a large sample of Mexican adults that generally represents the population of the country, 2) to identify the association between participants' weight and HRQoL and work productivity, and 3) to describe the weight loss methods currently reported by the sample.

\section{Materials and methods}

\section{Sample and procedure}

Data were collected using a cross-sectional survey of adults, aged 18 years or older, in Mexico $(\mathrm{N}=2,511)$. The only exclusion criterion was that participants had to be of a normal BMI $\left(18.5 \mathrm{~kg} / \mathrm{m}^{2}\right)$ or higher. Potential respondents were identified from an Internet panel, with some offline recruitment to ensure representation in all demographic strata.
Specifically, the Internet panel recruits members $(n=2,011)$ through various ways including e-newsletters and banner advertisement placements. Membership is optin, and those on the panel are provided the opportunity to complete periodic surveys on a variety of topics. Although the recruitment of panel members is not done purely by convenience sampling (i.e., some attempt is made to align with the demographic characteristics of the adult population), panel membership does skew toward the younger and more educated adults. To mitigate this bias, sampling for the present study was conducted using random stratification. Specifically, the characteristics of the Mexican adult population, with respect to age and sex, were identified using the International Database of the United States Census, ${ }^{36}$ which acquires information from governmental and statistical bureaus from each country in the world, and was selected to derive a sample that is a close approximation to the population of Mexico. These age and sex proportions were mimicked during the email recruitment of this study. Recruiting participants through Internet panels is increasingly used to enroll large numbers of diverse participants. For example, the approach was used in the development of the US National Institute of Health's roadmap initiative, Patient-Reported Outcomes Measurement Information System. ${ }^{37}$ It is also used to enroll participants in the National Health and Wellness Survey, which has been used to quantify the effect of chronic medical and psychiatric conditions on a range of measures. ${ }^{38-42}$

Since Internet panels underrepresent the elderly and the economically disadvantaged, a small subset of respondents were recruited through street intercepts $(n=79)$ to ensure appropriate representation of this subgroup. After ensuring eligibility, these respondents were brought into a centralized facility to complete the survey at a computer. The survey experience (e.g., questions, format) was identical to that of those who participated online.

To ensure that the sample size was adequate for performing the burden-of-obesity analysis in a subgroup of T2DM participants, an additional $\mathrm{N}=500$ respondents with the condition were recruited. Therefore, certain analyses focused on the general population ( $\mathrm{N}=2,011)$, and other burden-of-obesity analyses focused on everyone $(\mathrm{N}=2,511)$.

The study protocol was reviewed and granted exemption status by an independent Institutional Review Board (Pearl IRB; www.pearlirb.com) because de-identified data were collected which did not include responses outside the research that could reasonably place the subjects at risk of criminal or civil liability or be damaging to the financial standing, employability, or reputation of the subjects. All study materials, including the informed consent, were pro- 
vided in Spanish. Completion of survey was deemed to be agreement of consent from the participants for this study and participants who completed the survey were provided a small honorarium for participating.

\section{Measures \\ Demographics}

Sex, age, marital status, employment, and education were assessed for all respondents. Based on an algorithm from the Mexican Association of Marketing Research and Public Opinion Agencies, ${ }^{15}$ socioeconomic status (SES) was estimated from questions on household possessions (e.g., number of cars, bathrooms). The levels were $\mathrm{AB}, \mathrm{C}+, \mathrm{C}, \mathrm{C}-, \mathrm{D}+, \mathrm{D}$, and $\mathrm{E}$, in descending order of SES. An AB respondent, for example, would represent someone with a graduate degree, a large house (multiple bathrooms/bedrooms), and multiple automobiles, and an E respondent would represent, for example, someone with less than a primary school education, a house with 4 or fewer rooms, and no automobile.

\section{Health history and habits}

Respondents provided information on their height and weight. These variables were converted into a BMI value and then coded categorically as follows: normal weight (BMI $\geq 18.5-<25 \mathrm{~kg} / \mathrm{m}^{2}$ ), overweight (BMI $\geq 25-<30 \mathrm{~kg} / \mathrm{m}^{2}$ ), obese class I (BMI $\left.\geq 30-<35 \mathrm{~kg} / \mathrm{m}^{2}\right)$, obese class II (BMI $\geq 35-<40$ $\mathrm{kg} / \mathrm{m}^{2}$ ), and obese class III (BMI $\geq 40 \mathrm{~kg} / \mathrm{m}^{2}$ ). Underweight respondents $\left(\mathrm{BMI}<18.5 \mathrm{~kg} / \mathrm{m}^{2}\right)$ were not included in the study. Smoking history (current vs. former vs. never smoker), frequency of alcohol use, and the number of days exercised in the past month were also assessed.

\section{Comorbidities}

Respondents self-reported whether they had been diagnosed with the comorbidities associated with the 2008 version of the Charlson Comorbidity Index (CCI). ${ }^{43}$ A CCI score was generated for each respondent. Demographic and health history information was used to calculate a Diabetes Screening Score (DSS) value, ${ }^{44}$ which has been used to identify both patients with undiagnosed diabetes and those who have a high risk for developing diabetes in the future, the latter representing prediabetes for the purposes of our study. A self-reported diagnosis of hypertension or self-reported systolic and diastolic blood pressure values above $140 \mathrm{~mm} \mathrm{Hg}$ and $90 \mathrm{~mm} \mathrm{Hg}$, respectively, were used to classify respondents as having hypertension.

\section{Weight loss}

All respondents were asked whether they were currently taking steps to lose weight. Those who answered affirmatively were asked how much weight they had lost or gained in the past 6 months. Additionally, they were asked all the different weight loss methods they had used from a set list (e.g., exercise, dieting, prescription medications) along with their level of satisfaction with each and the associated monthly out-of-pocket costs for each.

\section{Health outcomes}

HRQoL was assessed using the visual analog scale (VAS) and health utilities from the EuroQoL-5D (EQ-5D)-3L. ${ }^{45}$ The Work Productivity and Activity Impairment (WPAI) questionnaire was included to assess the level of absenteeism (i.e., percentage of work missed due to health), presenteeism (i.e., the percentage of work rendered ineffective due to health), overall work impairment (i.e., the total percentage of work missed due to either absenteeism or presenteeism), and activity impairment (i.e., the percentage of daily activities that were rendered ineffective due to health) experienced in the past 7 days due to health-related reasons. ${ }^{46}$ The WPAI questionnaire was only answered by those currently employed. Finally, the number of health care provider visits, emergency room (ER) visits, and hospitalizations, in the past 6 months, was also queried.

\section{Statistical analysis \\ Burden-of-obesity analyses}

Among the general population subsample $(n=2,011)$, the sample prevalence of T2DM, prediabetes, normoglycemia, and cardiovascular comorbidities (i.e., congestive heart failure, myocardial infarction, peripheral vascular disease, hypertension, warfarin use) was derived.

Next, the association between obesity and health outcomes was examined among all respondents who were classified as normal weight, overweight, obese class I, obese class II, or obese class III. Respondents who were underweight were excluded from these analyses. Differences among respondents with varying levels of BMI categories were made with respect to demographics, health history, and health outcome variables to assess for potential covariates to include in subsequent modeling. To boost sample sizes, obese class II and obese class III categories were combined. Chi-square tests and one-way analyses of variance were used for categorical and continuous outcomes, respectively. Generalized linear models (GLMs) were used to predict health outcomes from BMI categories controlling for sex, age, marital status, education, exercise frequency, and the CCI. These analyses were replicated among those with T2DM, prediabetes, and hypertension. 
To convert work productivity loss to indirect costs, the adjusted mean level of overall work productivity loss for each BMI category, derived from the GLMs, was multiplied by the average wage in Mexico (12,850 Mexican pesos $[\mathrm{MXN}]^{47}$ ), as outlined in previous research. ${ }^{48}$

Because certain antidepressants, such as some selective serotonin reuptake inhibitors, can increase BMI, and those conditions can also directly affect health outcomes, additional sensitivity analyses were conducted controlling for self-reported depression. Depression is part of the $2008 \mathrm{CCI}$ calculation that was used for this study, ${ }^{43}$ but it was included instead as a separate variable in these sensitivity analyses.

\section{Treatment patterns of weight loss}

Among those taking steps to lose weight, the treatment patterns of weight loss were examined descriptively (i.e., frequencies and percentages were reported for categorical variables, and means and SDs were reported for continuous variables).

\section{Results \\ Descriptive statistics}

The overall sample included about the same number of men $(50.62 \%)$ and women $(49.38 \%)$ with a mean age of 40.66 years $(\mathrm{SD}=14.50)$. The SES was relatively high; $58.1 \%$ had attained a university degree, and $40.62 \%$ belonged to the highest SES level (AB) based on household possessions. The distribution of BMI categories was as follows: underweight $=35(1.39 \%)$, normal weight $=901(35.88 \%)$, overweight $=962(38.31 \%)$, obese class $\mathrm{I}=425(16.93 \%)$, obese class $\mathrm{II}=132(5.26 \%)$, and obese class $\mathrm{III}=56(2.23 \%)$.

\section{Comorbidity burden of obesity}

A total of 826 respondents $(32.90 \%)$ reported having T2DM, 900 respondents $(35.84 \%)$ reported having hypertension, and $454(18.08 \%)$ reported both conditions. Based on the DSS, 425 respondents (16.93\%) were classified as having prediabetes.

\section{Health outcome burden of obesity}

Several demographic differences were observed across BMI category (Table 1). Although males were more likely to have a BMI $\geq 25$ and $<30$, females were more likely to have a BMI $\geq 30$ ( $P<0.05)$. Increasing BMI was associated increasing age, an increased likelihood of being married/living with a partner, and not having a university degree (all $P<0.05$ ). Increasing BMI was also associated with fewer days exercised in the past month and a higher $\mathrm{CCI}$ (both $P<0.05$ ). No significant differences were observed with respect to SES, smoking behavior, or frequency of alcohol intake.

Controlling for demographic and health history differences among BMI categories, respondents in overweight, obese class I, and obese class II-III categories all reported significantly lower EQ-5D VAS and EQ-5D health utility scores, and greater impairment in daily activities compared with respondents in the normal weight category (Table 2). Respondents in the obese class I and obese class II-III categories reported greater levels of presenteeism, overall work impairment, and health care provider visits, compared with normal weight respondents (all $P<0.05$ ). Respondents in the obese class I category reported significantly more ER visits than normal weight respondents $(P<0.05)$. No other differences were observed.

The same relationships between BMI and health outcomes were explored among those with T2DM, prediabetes, and hypertension (Table 3). Although these subgroup analyses were underpowered, respondents in obese class I and combined obese class II-III categories reported significantly lower health utilities than respondents in the normal weight category for all comorbidity subgroups. The results for work productivity and health care resource use were more inconsistent. Only those respondents with prediabetes in the obese class II-III category reported significantly greater presenteeism and overall work impairment compared with normal weight respondents. Respondents who were in obesity class I category and were either in the T2DM or hypertension groups reported more ER visits in the past 6 months than their normal weight counterparts. Those respondents with T2DM, and separately hypertension, in the obese class I category reported more ER visits in the past 6 months than normal weight respondents. Indirect costs rose as BMI increased, with the costs rising from 2,422 MXN (normal weight) to 2,614 MXN (overweight) to 2,993 MXN (obese class I) to 3,317 MXN (obese class II-III).

The sensitivity analysis controlling for depression included as a separate variable rather than being included as part of the CCI found that adjusted means of the two models differed by less than a tenth of a point indicating that depression was not an integral component of BMI.

\section{Management of obesity}

Of the total number of respondents, $62.17 \%(n=1,561)$ were taking steps to lose weight, and the primary reason for doing so was to improve their health $(60.79 \%)$. Despite these intentions, success was limited. Only slightly more than a third $(34.34 \%$ ) reported having lost weight in the past 6 months 
Table I Demographic and health history differences across BMI categories $(N=2,476)$

\begin{tabular}{|c|c|c|c|c|c|}
\hline & \multicolumn{4}{|l|}{ BMI category } & \multirow[t]{2}{*}{$P$ value } \\
\hline & $\begin{array}{l}\text { Normal weight } \\
(18.5-<25)(n=901)\end{array}$ & $\begin{array}{l}\text { Overweight } \\
(25-<30)(n=962)\end{array}$ & $\begin{array}{l}\text { Obese class I } \\
(30-<35)(n=425)\end{array}$ & $\begin{array}{l}\text { Obese class II-III } \\
(35+)(n=188)\end{array}$ & \\
\hline Sex & & & & & $<0.001$ \\
\hline Male, n (\%) & 422 (46.84\%) & $548(56.96 \%)$ & 199 (46.82\%) & $92(48.94 \%)$ & \\
\hline Female, n (\%) & $479(53.16 \%)$ & $414(43.04 \%)$ & $226(53.18 \%)$ & $96(51.06 \%)$ & \\
\hline Age & & & & & $<0.001$ \\
\hline Mean \pm SD & $37.52 \pm 14.06$ & $42.05 \pm|4.4|$ & $43.86 \pm 14.67$ & $42.82 \pm 13.68$ & \\
\hline Marital status & & & & & $<0.001$ \\
\hline Single, n (\%) & $4 \mathrm{II}(45.62 \%)$ & 338 (35.14\%) & 136 (32.00\%) & 61 (32.45\%) & \\
\hline Married/living with partner, n (\%) & $490(54.38 \%)$ & $624(64.86 \%)$ & $289(68.00 \%)$ & 127 (67.55\%) & \\
\hline Employment status & & & & & 0.455 \\
\hline Not currently employed, n (\%) & $226(25.08 \%)$ & $218(22.66 \%)$ & 105 (24.7I\%) & 51 (27.13\%) & \\
\hline Employed, $\mathrm{n}(\%)$ & 675 (74.92\%) & 744 (77.34\%) & $320(75.29 \%)$ & 137 (72.87\%) & \\
\hline Highest level of education & & & & & 0.040 \\
\hline Less than university degree, $\mathrm{n}(\%)$ & 359 (39.84\%) & 389 (40.44\%) & 197 (46.35\%) & $87(46.28 \%)$ & \\
\hline University degree, n (\%) & $542(60.16 \%)$ & $573(59.56 \%)$ & $228(53.65 \%)$ & I0I (53.72\%) & \\
\hline Socioeconomic status & & & & & 0.884 \\
\hline$A B, n(\%)$ & 367 (40.73\%) & 394 (40.96\%) & 166 (39.06\%) & 77 (40.96\%) & \\
\hline $\mathrm{C}+, \mathrm{n}(\%)$ & 297 (32.96\%) & $328(34.10 \%)$ & $15 \mathrm{I}(35.53 \%)$ & $60(31.91 \%)$ & \\
\hline $\mathrm{C}, \mathrm{n}(\%)$ & $129(14.32 \%)$ & $122(12.68 \%)$ & $54(12.71 \%)$ & $23(12.23 \%)$ & \\
\hline $\mathrm{C}-, \mathrm{n}(\%)$ & $62(6.88 \%)$ & $66(6.86 \%)$ & $35(8.24 \%)$ & $15(7.98 \%)$ & \\
\hline $\mathrm{D}+, \mathrm{n}(\%)$ & $37(4.11 \%)$ & 39 (4.05\%) & $15(3.53 \%)$ & 9 (4.79\%) & \\
\hline $\mathrm{D}, \mathrm{n}(\%)$ & $9(1.00 \%)$ & $13(1.35 \%)$ & $4(0.94 \%)$ & $4(2.13 \%)$ & \\
\hline \multicolumn{6}{|l|}{ Health behaviors } \\
\hline Smoking behavior & & & & & 0.274 \\
\hline Current smoker, n (\%) & 286 (31.74\%) & $269(27.96 \%)$ & I IO (25.88\%) & $54(28.72 \%)$ & \\
\hline Former smoker, $\mathrm{n}(\%)$ & 327 (36.29\%) & $393(40.85 \%)$ & $|8|$ (42.59\%) & $74(39.36 \%)$ & \\
\hline Never smoker, n (\%) & $288(31.96 \%)$ & $300(31.19 \%)$ & 134 (31.53\%) & $60(31.91 \%)$ & \\
\hline Days exercised in the past month & & & & & $<0.001$ \\
\hline Mean \pm SD & $9.92 \pm 9.15$ & $8.93 \pm 8.89$ & $7.18 \pm 8.18$ & $6.7 I \pm 8.04$ & \\
\hline Alcohol use frequency & & & & & 0.083 \\
\hline Daily, n (\%) & $7(0.78 \%)$ & $16(1.66 \%)$ & $2(0.47 \%)$ & $0(0.00 \%)$ & \\
\hline 4-6 times a week, n (\%) & $17(1.89 \%)$ & $17(1.77 \%)$ & $7(1.65 \%)$ & $3(1.60 \%)$ & \\
\hline 2-3 times a week, n (\%) & 79 (8.77\%) & $75(7.80 \%)$ & $21(4.94 \%)$ & $12(6.38 \%)$ & \\
\hline Once a week, n (\%) & $152(16.87 \%)$ & $164(17.05 \%)$ & $76(17.88 \%)$ & $25(13.30 \%)$ & \\
\hline 2-3 times a month, $\mathrm{n}(\%)$ & 144 (15.98\%) & $15 \mid(15.70 \%)$ & $60(14.12 \%)$ & $23(12.23 \%)$ & \\
\hline Once a month or less often, $\mathrm{n}(\%)$ & 297 (32.96\%) & 307 (31.91\%) & 133 (31.29\%) & 64 (34.04\%) & \\
\hline I do not drink alcohol, n (\%) & 205 (22.75\%) & $232(24.12 \%)$ & $126(29.65 \%)$ & $61(32.45 \%)$ & \\
\hline Charlson Comorbidity Index & & & & & $<0.001$ \\
\hline Mean \pm SD & $0.94 \pm 2.34$ & $\mathrm{I} .14 \pm 1.85$ & $1.68 \pm 2.13$ & $2.03 \pm 2.26$ & \\
\hline \multicolumn{6}{|l|}{ Comorbidities } \\
\hline T2DM, n (\%) & 204 (24.91\%) & $344(42.00 \%)$ & 163 (19.90\%) & $108(13.19 \%)$ & $<0.001$ \\
\hline Prediabetes, n (\%) & $39(9.18 \%)$ & I 72 (40.47\%) & 163 (38.35\%) & $5 \mathrm{I}(12.00 \%)$ & $<0.001$ \\
\hline Hypertension, n (\%) & $229(25.59 \%)$ & $328(36.65 \%)$ & $217(9.27 \%)$ & 121 (I3.52\%) & $<0.001$ \\
\hline
\end{tabular}

Abbreviations: BMI, body mass index; T2DM, type 2 diabetes mellitus.

(43.31\% reported gaining weight), and the mean weight change was $0.47 \mathrm{~kg}(\mathrm{SD}=7.33)$.

The most common treatments used for weight loss included exercise and dieting (Table 4). Of the total number of respondents, $27.55 \%$ had used an herbal product, $19.03 \%$ used over-the-counter (OTC) orlistat weight loss pills $(60 \mathrm{mg})$, and $17.10 \%$ used a prescription medication (the most common prescription medication was orlistat
[120 mg], which was used by $46.44 \%$ of those who were using a prescription medication). However, discontinuation rates were high with these treatments. Of those who ever used either OTC or prescription medications, less than half were currently using those methods.

For those using OTC and prescription medications $(\mathrm{N}=345)$, the current out-of-pocket costs were $528.58 \mathrm{MXN}$ $(\mathrm{SD}=658.00$; median=350.00). Few differences in out-of- 
Table 2 Regression-adjusted health outcome differences across BMI categories $(\mathrm{N}=2,476)$

\begin{tabular}{|c|c|c|c|c|c|c|}
\hline Dependent variable & BMI group & $\begin{array}{l}\text { Adjusted } \\
\text { mean }\end{array}$ & SE & $95 \%$ LCL & 95\% UCL & $P$ value \\
\hline EQ-5D: VAS & Overweight $(25-30)$ & 81.85 & 0.38 & 81.09 & 82.60 & $<0.000$ I \\
\hline EQ-5D: VAS & Obese class I $(30-<35)$ & 79.62 & 0.58 & 78.48 & 80.77 & $<0.0001$ \\
\hline EQ-5D: VAS & Obese class II-III (35+) & 77.33 & 0.87 & 75.62 & 79.05 & $<0.0001$ \\
\hline EQ-5D: VAS & Normal weight $(18.5-<25)$ & 84.23 & 0.40 & 83.44 & 85.02 & \\
\hline EQ-5D: health utility & Overweight $(25-30)$ & 0.83 & 0.00 & 0.82 & 0.84 & 0.0187 \\
\hline EQ-5D: health utility & Obese class I $(30-<35)$ & 0.82 & 0.01 & 0.80 & 0.83 & 0.0003 \\
\hline EQ-5D: health utility & Obese class II-III (35+) & 0.78 & 0.01 & 0.76 & 0.80 & $<0.0001$ \\
\hline EQ-5D: health utility & Normal weight $(18.5-<25)$ & 0.85 & 0.01 & 0.84 & 0.86 & \\
\hline Absenteeism \% (WPAI-GH) & Overweight $(25-<30)$ & 5.05 & 0.52 & 4.13 & 6.17 & 0.5533 \\
\hline Absenteeism \% (WPAI-GH) & Obese class I $(30-<35)$ & 4.66 & 0.73 & 3.42 & 6.33 & 0.9695 \\
\hline Absenteeism \% (WPAI-GH) & Obese class II-III (35+) & 5.29 & 1.27 & 3.31 & 8.48 & 0.6097 \\
\hline Absenteeism \% (WPAI-GH) & Normal weight $(18.5-<25)$ & 4.62 & 0.50 & 3.74 & 5.71 & \\
\hline Presenteeism \% (WPAI-GH) & Overweight $(25-<30)$ & 17.65 & 0.91 & 15.95 & 19.53 & 0.2509 \\
\hline Presenteeism \% (WPAI-GH) & Obese class I $(30-<35)$ & 20.94 & 1.65 & 17.94 & 24.44 & 0.0080 \\
\hline Presenteeism \% (WPAI-GH) & Obese class II-III (35+) & 23.24 & 2.81 & 18.34 & 29.45 & 0.0069 \\
\hline Presenteeism \% (WPAI-GH) & Normal weight $(18.5-<25)$ & 16.18 & 0.89 & 14.53 & 18.02 & \\
\hline Overall work impairment \% (WPAI-GH) & Overweight $(25-<30)$ & 20.34 & 0.99 & 18.48 & 22.39 & 0.2898 \\
\hline Overall work impairment \% (WPAI-GH) & Obese class I $(30-<35)$ & 23.29 & 1.74 & 20.11 & 26.97 & 0.0219 \\
\hline Overall work impairment \% (WPAI-GH) & Obese class II-III (35+) & 25.81 & 2.95 & 20.62 & 32.30 & 0.0134 \\
\hline Overall work impairment \% (WPAI-GH) & Normal weight $(18.5-<25)$ & 18.85 & 0.98 & 17.02 & 20.88 & \\
\hline Activity impairment \% (WPAI-GH) & Overweight $(25-<30)$ & 18.31 & 0.89 & 16.64 & 20.14 & 0.0380 \\
\hline Activity impairment \% (WPAI-GH) & Obese class I $(30-<35)$ & 20.83 & 1.54 & 18.01 & 24.09 & 0.0023 \\
\hline Activity impairment \% (WPAl-GH) & Obese class II-III (35+) & 24.82 & 2.82 & 19.87 & 31.00 & 0.0003 \\
\hline Activity impairment \% (WPAI-GH) & Normal weight $(18.5-<25)$ & 15.78 & 0.82 & 14.26 & 17.46 & \\
\hline Number of provider visits (past 6 months) & Overweight $(25-<30)$ & 3.22 & 0.11 & 3.02 & 3.44 & 0.4151 \\
\hline Number of provider visits (past 6 months) & Obese class I $(30-<35)$ & 3.40 & 0.17 & 3.08 & 3.75 & 0.1319 \\
\hline Number of provider visits (past 6 months) & Obese class II-III (35+) & 3.73 & 0.27 & 3.23 & 4.31 & 0.0235 \\
\hline Number of provider visits (past 6 months) & Normal weight $(18.5-<25)$ & 3.09 & 0.11 & 2.88 & 3.32 & \\
\hline Number of ER visits (past 6 months) & Overweight $(25-<30)$ & 0.29 & 0.03 & 0.24 & 0.34 & 0.1414 \\
\hline Number of ER visits (past 6 months) & Obese class I $(30-<35)$ & 0.35 & 0.05 & 0.28 & 0.46 & 0.0126 \\
\hline Number of ER visits (past 6 months) & Obese class II-III (35+) & 0.26 & 0.05 & 0.18 & 0.39 & 0.6042 \\
\hline Number of ER visits (past 6 months) & Normal weight $(18.5-<25)$ & 0.24 & 0.02 & 0.19 & 0.29 & \\
\hline Number of hospitalizations (past 6 months) & Overweight $(25-<30)$ & 0.17 & 0.02 & 0.14 & 0.21 & 0.3924 \\
\hline Number of hospitalizations (past 6 months) & Obese class I $(30-<35)$ & 0.22 & 0.03 & 0.17 & 0.30 & 0.4247 \\
\hline Number of hospitalizations (past 6 months) & Obese class II-III (35+) & 0.22 & 0.05 & 0.14 & 0.33 & 0.6233 \\
\hline Number of hospitalizations (past 6 months) & Normal weight $(18.5-<25)$ & 0.19 & 0.02 & 0.15 & 0.24 & \\
\hline
\end{tabular}

Notes: All models were controlled for sex, age, marital status, education, exercise frequency, and the CCl. $P<0.05$.

Abbreviations: BMI, body mass index; SE, standard error; LCL, lower confidence limit; UCL, upper confidence limit; EQ-5D, EuroQoL-5D; VAS, visual analog scale; WPAI$\mathrm{GH}$, Work Productivity and Activity Impairment - General Health; ER, emergency room; CCl, Charlson Comorbidity Index.

pocket costs were observed by demographic and health history variables, though small sample sizes precluded statistical comparisons. Out-of-pocket costs trended lower among the elderly and higher among those with comorbidities (i.e., T2DM, hypertension, CCI score of $>1$ ).

\section{Discussion}

In a sample of Mexican adults, who were recruited to reflect the overall sex and age of the population, it was found that
$62 \%$ were, at least, overweight. Heavier respondents reported higher comorbidity burden, as well as worsening HRQoL and work productivity. Further, a large proportion of the sample is actively trying to lose weight. Taken together, these findings indicate that many Mexicans are experiencing the deleterious effects associated with BMI despite the desire to lose weight.

These findings are comparable to past research, ${ }^{1}$ though our study and the high comorbidity burden associated with increasing BMI is consistent with other studies too. ${ }^{1,2,6}$ 
Table 3 Regression-adjusted health outcome differences across BMI categories among those with T2DM, prediabetes, and hypertension

\begin{tabular}{|c|c|c|c|c|c|c|c|c|c|c|}
\hline \multirow{2}{*}{$\begin{array}{l}\text { Dependent } \\
\text { variable }\end{array}$} & \multirow[t]{2}{*}{ BMI group } & \multicolumn{3}{|c|}{ T2DM $(n=819)$} & \multicolumn{3}{|c|}{ Prediabetes $(n=425)$} & \multicolumn{3}{|c|}{ Hypertension $(n=825)$} \\
\hline & & $\begin{array}{l}\text { Adjusted } \\
\text { mean }\end{array}$ & $95 \% \mathrm{Cl}$ & $P$ value & $\begin{array}{l}\text { Adjusted } \\
\text { mean }\end{array}$ & $95 \% \mathrm{Cl}$ & $P$ value & $\begin{array}{l}\text { Adjusted } \\
\text { mean }\end{array}$ & $95 \% \mathrm{Cl}$ & $P$ value \\
\hline \multirow[t]{4}{*}{ EQ-5D: VAS } & Overweight $(25-<30)$ & 78.07 & $76.71-79.42$ & 0.8418 & 82.64 & $79.26-86.02$ & 0.6453 & 78.06 & $76.66-79.46$ & 0.0008 \\
\hline & Obese class I $(30-<35)$ & 75.98 & $74.00-77.96$ & 0.090 & 79.79 & $76.30-83.28$ & 0.0898 & 77.06 & 75.34-78.78 & 0.0001 \\
\hline & Obese class II-III (35+) & 75.48 & 73.08-77.87 & 0.0649 & 76.66 & $72.26-81.06$ & 0.0108 & 74.95 & $72.65-77.26$ & $<.0001$ \\
\hline & Normal weight $(18.5-<25)$ & 78.29 & $76.52-80.07$ & & 83.65 & $78.85-88.44$ & & 81.84 & $80.15-83.54$ & \\
\hline \multirow{4}{*}{$\begin{array}{l}\text { EQ-5D: health } \\
\text { utility }\end{array}$} & Overweight $(25-30)$ & 0.80 & $0.79-0.82$ & 0.5873 & 0.84 & $0.79-0.88$ & 0.5398 & 0.79 & $0.78-0.81$ & 0.1221 \\
\hline & Obese class I $(30-<35)$ & 0.77 & $0.75-0.79$ & 0.0172 & 0.83 & $0.78-0.87$ & 0.353 & 0.77 & $0.75-0.80$ & 0.0079 \\
\hline & Obese class II-III (35+) & 0.76 & $0.73-0.79$ & 0.0043 & 0.76 & $0.7 I-0.8 I$ & 0.0067 & 0.74 & $0.72-0.77$ & $<.0001$ \\
\hline & Normal weight $(18.5-<25)$ & 0.81 & $0.79-0.83$ & & 0.85 & $0.79-0.91$ & & 0.81 & $0.79-0.84$ & \\
\hline \multirow[t]{4}{*}{ Absenteeism \% } & Overweight $(25-<30)$ & 7.86 & $6.10-10.15$ & 0.8587 & - & - & - & 7.11 & $5.52-9.16$ & 0.3639 \\
\hline & Obese class I $(30-<35)$ & 7.38 & $5.09-10.7 \mid$ & 0.6908 & - & - & - & 6.71 & $4.91-9.18$ & 0.5701 \\
\hline & Obese class II-III (35+) & 8.06 & $5.07-12.79$ & 0.9644 & - & - & - & 5.81 & $3.77-8.95$ & 0.9525 \\
\hline & Normal weight $(18.5-<25)$ & 8.16 & $5.91-11.28$ & & - & - & - & 5.91 & $4.36-8.01$ & \\
\hline \multirow{4}{*}{$\begin{array}{l}\text { Presenteeism } \\
\%\end{array}$} & Overweight $(25-<30)$ & 22.97 & $20-26.37$ & 0.4691 & 15.04 & $8.49-26.65$ & 0.3674 & 23.39 & $20.4-26.81$ & 0.6156 \\
\hline & Obese class I $(30-<35)$ & 28.24 & $23.05-34.59$ & 0.3719 & 21.33 & $\mid 1.81-38.53$ & 0.0614 & 27.48 & $23.2-32.54$ & 0.075 \\
\hline & Obese class II-III (35+) & 26.59 & $20.64-34.27$ & 0.6934 & 39.12 & $18.16-84.3$ & 0.0033 & 28.14 & $22.24-35.6$ & 0.1067 \\
\hline & Normal weight $(18.5-<25)$ & 24.96 & $20.87-29.85$ & & 10.99 & $5.19-23.3$ & & 22.13 & $18.77-26.1$ & \\
\hline \multirow{4}{*}{$\begin{array}{l}\text { Overall work } \\
\text { impairment \% }\end{array}$} & Overweight $(25-<30)$ & 26.62 & $23.44-30.23$ & 0.2985 & 18.48 & $10.51-32.5$ & 0.2601 & 27.17 & $23.89-30.9$ & 0.5143 \\
\hline & Obese class I $(30-<35)$ & 31.55 & $26.17-38.03$ & 0.6386 & 24.6 & $13.69-44.18$ & 0.0548 & 30.66 & $26.15-35.94$ & 0.0999 \\
\hline & Obese class II-III (35+) & 30.49 & $24.15-38.5$ & 0.8618 & 40.14 & $|8.8|-85.69$ & 0.0063 & 31.4 & $25.17-39.16$ & 0.1299 \\
\hline & Normal weight $(18.5-<25)$ & 29.72 & $25.21-35.04$ & & 12.49 & $5.95-26.21$ & & 25.4 & $21.75-29.66$ & \\
\hline \multirow{4}{*}{$\begin{array}{l}\text { Activity } \\
\text { impairment \% }\end{array}$} & Overweight $(25-<30)$ & 23.94 & $21.12-27.13$ & 0.8742 & 15.12 & $8.42-27.17$ & $0.474 I$ & 23.40 & $20.61-26.56$ & 0.5419 \\
\hline & Obese class I $(30-<35)$ & 28.88 & $24.09-34.62$ & 0.0982 & 15.84 & $8.8-28.51$ & 0.4129 & 28.30 & $24.22-33.07$ & 0.0247 \\
\hline & Obese class II-III (35+) & 30.25 & $24.12-37.93$ & 0.0793 & 28.87 & $|3.59-6| .33$ & $0.041 \mathrm{I}$ & 32.38 & $26.09-40.18$ & 0.0047 \\
\hline & Normal weight $(18.5-<25)$ & 23.55 & $20.09-27.61$ & & 11.69 & $5.4-25.3$ & & 21.99 & $18.9-25.6$ & \\
\hline \multirow{4}{*}{$\begin{array}{l}\text { Number of } \\
\text { provider visits }\end{array}$} & Overweight $(25-<30)$ & 4.21 & $3.84-4.62$ & 0.124 & 2.75 & $2.14-3.54$ & $0.7 \mid 35$ & 3.96 & $3.6-4.37$ & 0.5938 \\
\hline & Obese class I $(30-<35)$ & 4.87 & $4.27-5.55$ & 0.7715 & 2.84 & $2.21-3.66$ & 0.5945 & 4.13 & $3.68-4.65$ & 0.9957 \\
\hline & Obese class II-III (35+) & 4.79 & $4.09-5.62$ & 0.9137 & 3.12 & $2.26-4.3$ & 0.3667 & 4.46 & $3.82-5.21$ & 0.4361 \\
\hline & Normal weight $(18.5-<25)$ & 4.74 & $4.21-5.34$ & & 2.59 & I.8-3.73 & & 4.13 & $3.68-4.64$ & \\
\hline \multirow{4}{*}{$\begin{array}{l}\text { Number of ER } \\
\text { visits }\end{array}$} & Overweight $(25-<30)$ & 0.42 & $0.33-0.55$ & 0.9373 & 0.16 & $0.07-0.37$ & 0.5282 & 0.46 & $0.36-0.6$ & 0.1387 \\
\hline & Obese class I $(30-<35)$ & 0.72 & $0.52-0.98$ & 0.0271 & 0.23 & $0.1-0.53$ & 0.2616 & 0.58 & $0.43-0.78$ & 0.0161 \\
\hline & Obese class II-III (35+) & $0.4 \mathrm{I}$ & $0.27-0.63$ & 0.8506 & 0.22 & $0.08-0.6 \mathrm{I}$ & 0.3459 & 0.34 & $0.21-0.52$ & 0.97 \\
\hline & Normal weight $(18.5-<25)$ & 0.43 & $0.31-0.6$ & & 0.10 & $0.02-0.42$ & & 0.34 & $0.24-0.47$ & \\
\hline \multirow{4}{*}{$\begin{array}{l}\text { Number of } \\
\text { hospitalizations }\end{array}$} & Overweight $(25-<30)$ & 0.27 & $0.2-0.35$ & 0.0366 & 0.11 & $0.05-0.28$ & 0.2057 & 0.29 & $0.21-0.39$ & 0.765 \\
\hline & Obese class I $(30-<35)$ & 0.49 & $0.36-0.68$ & 0.3783 & 0.14 & $0.05-0.36$ & 0.328 & 0.36 & $0.26-0.5 \mathrm{I}$ & 0.5188 \\
\hline & Obese class II-III (35+) & 0.31 & $0.2-0.48$ & 0.3045 & 0.19 & $0.06-0.6$ & 0.6293 & 0.28 & $0.17-0.46$ & 0.7473 \\
\hline & Normal weight $(18.5-<25)$ & 0.41 & $0.3-0.55$ & & 0.27 & $0.07-1.03$ & & 0.31 & $0.22-0.44$ & \\
\hline
\end{tabular}

Notes: All models were controlled for sex, age, marital status, education, exercise frequency, and the $\mathrm{CCl}$. The absenteeism model failed to converge in the prediabetes subsample. $P<0.05$. '-' indicates non-convergence.

Abbreviations: BMI, body mass index; T2DM, type 2 diabetes mellitus; EQ-5D EuroQoL-5D; VAS, visual analog scale; ER, emergency room; CCl, Charlson Comorbidity Index.

Indeed, $75.2 \%$ of individuals with a BMI $\geq 35$ had either T2DM (29.9\%) or prediabetes (45.3\%). Additionally, patientreported participants reported more impaired HRQoL and work productivity as burden of BMI increases. The strongest effects were observed with HRQoL. Across the sample, each BMI category was associated with a statistically and 
Table 4 Treatments ever and currently used by those taking steps to lose weight $(n=I, 56 I)$

\begin{tabular}{|c|c|c|c|c|}
\hline & \multicolumn{2}{|c|}{ Ever used } & \multicolumn{2}{|c|}{ Currently using } \\
\hline & $\mathbf{n}$ & $\%$ & $\mathbf{n}$ & $\%$ \\
\hline Exercise & 1,246 & 79.82 & 994 & 79.78 \\
\hline Dieting & 1,023 & 65.53 & 808 & 78.98 \\
\hline Herbal products & 430 & 27.55 & 210 & 48.84 \\
\hline Consulting a specialist & 429 & 27.48 & 189 & 44.06 \\
\hline Gym memberships & 397 & 25.43 & 161 & 40.55 \\
\hline Diet supplements & 375 & 24.02 & 155 & 41.33 \\
\hline OTC $60 \mathrm{mg}$ orlistat & 297 & 19.03 & 83 & 27.95 \\
\hline Prescription medication & 267 & 17.10 & 92 & 34.46 \\
\hline Redustat (I 20 mg orlistat) & 124 & 46.44 & 36 & 29.03 \\
\hline $\begin{array}{l}\text { Redotex (atropine+norpseudo } \\
\text { ephedrine+diazepam+triiodoth } \\
\text { yronine) }\end{array}$ & 90 & 33.71 & 27 & 30.00 \\
\hline Solucaps (mazindol) & 46 & 17.23 & 11 & 23.91 \\
\hline Lindeza (I 20 mg orlistat) & 42 & 15.73 & 9 & 21.43 \\
\hline Asenlix (clobenzorex) & 38 & 14.23 & 7 & 18.42 \\
\hline Terfamez (phentermine) & 36 & 13.48 & 14 & 38.89 \\
\hline Obeclox (clobenzorex) & 22 & 8.24 & 5 & 22.73 \\
\hline Other prescription medication & 58 & 21.72 & 16 & 27.59 \\
\hline $\begin{array}{l}\text { Joining weight management } \\
\text { program }\end{array}$ & 90 & 5.77 & 29 & 32.22 \\
\hline Surgical bariatric procedure & 28 & 1.79 & 15 & 53.57 \\
\hline Other & 47 & 3.01 & 39 & 82.98 \\
\hline
\end{tabular}

Note: "Currently using" percentages are based on those who "ever used" the given method (e.g., 79.78\% of those who ever used exercise are currently using exercise). Abbreviation: OTC, over-the-counter.

clinically significant decrement in health utilities compared with normal weight respondents. Indeed, the HRQoL for those in the combined obese class II-III was worse than what has been reported for psoriasis, migraine, asthma, and glaucoma, and comparable to gout and cardiac arrhythmias. ${ }^{49}$ Effects on daily activities were also observed as a function of obesity. Respondents with obesity reported nearly double the level of work-related impairment, even after adjusting for demographic and health history confounds. The same pattern of results was observed for the T2DM, prediabetes, and hypertension subgroups.

Among the employed, greater overall work-related impairment was also observed with increasing BMI, but these associations were a function of increasing presenteeism as opposed to absenteeism. In other words, after accounting for demographic and health history characteristics, obesity was generally unrelated to an individual's ability to show up for work, but was related to the individual's productivity while working. Overall, a third to two-thirds greater work-related impairment was observed among those with obesity compared with normal weight respondents. This corresponded to an incremental 1,400 MXN per employee per year, a value which exceeds $10 \%$ of the average annual wage for an indi- vidual. Due to sample size restrictions for the employed group in other comorbidity subgroups, leading to wide confidence intervals, it is difficult to make firm conclusions on how the pattern may differ in these cases. Larger cohort studies are necessary to assess obesity-related indirect costs among those with T2DM, prediabetes, and hypertension.

Interestingly, few associations between obesity and health care resource utilization were observed. We attribute this partially to sample size. The variability in health care resource utilization was high, and even somewhat large differences in the mean number of visits between groups were not statistically significant. Another factor to consider is the cross-sectional design which may not fully capture the long-term implications that have previously been found with obesity. ${ }^{1,2,6,7,9-11}$ This is an area that warrants further research.

It should be noted that the effects of increasing BMI in our prediabetes sample were small, with the results resembling more the overall population than the T2DM population. In part, this may be due to the fact that this group was the smallest subgroup which limited statistical power. Another issue was that some of the covariates in the regression model (e.g., age, sex, and smoking) were used to define the presence of prediabetes. As a result, when controlling for these factors (which are independently associated with health outcomes), in part, we were controlling for the "severity" of prediabetes. This likely diminished our effects and points to future research where alternative methods of classifying the prediabetes patients for the purposes of burden-of-obesity analyses (e.g., using glycemic levels rather than patientreported health history) could be considered.

Over $60 \%$ of those in Mexico are taking steps to lose weight, which is much higher than the $40 \%$ reported in a previous study. ${ }^{35}$ Similarly, nearly $70 \%$ of those, without experience in using a prescription medication, expressed a willingness to do so in our study compared with $24-33 \%$ of obese respondents in the US. ${ }^{50}$ This higher level of interest does not seem to be explained by a greater BMI category. Our reported BMI distribution (e.g., overweight $=38 \%$, obese $=24 \%$ ) was less extreme than that reported in the US. . $^{50,51}$

However, regardless of these weight loss intentions, the success was limited, which is consistent with previous research in this domain. ${ }^{34}$ Overall weight change in the past 6 months was low, with more respondents actually gaining weight in the past 6 months than losing weight. Additionally, aside from diet and exercise, discontinuation rates for weight loss interventions were high, ranging from 50 to $70 \%$. Future research would be necessary to understand the reasons for the high discontinuation rates. 


\section{Limitations}

This study, like all others, has limitations. All data were self-reported, and no objective confirmation of weight, treatment use, or health care resource use was available. It was also cross-sectional, so it was not possible to assess a clear causal pathway between BMI/weight and health outcomes. Further, indirect costs were estimated based on estimated wage rates and may be different than true costs obtained through other datasets. Additionally, this study does not quantify all costs associated with obesity. Supplementary sources of information are needed to further calculate direct and other indirect costs.

Although the survey sample was demographically representative with respect to age and sex, there are a few limitations that pertain to the external validity of the findings. Most respondents were a member of an Internet panel and completed the survey online; thus, those without Internet access or otherwise economically disadvantaged might be underrepresented in the data. Finally, some caution should be applied when examining these results because small sample sizes in some of the analyses resulted in less statistical precision in these projections.

\section{Conclusion}

In summary, the results suggest a significant unmet need for the treatment of overweight and obese adults in Mexico. The majority of respondents were overweight, and the results suggest a significant opportunity to improve health outcomes, particularly HRQoL. The strongest effects were observed for those who reported being obese. This pattern was similar for respondents with $\mathrm{T} 2 \mathrm{DM}$, prediabetes, and hypertension, with increasing BMI category. Despite these effects, there is an apparent high degree of willingness for respondents to lose weight; however, the success of available interventions is limited, and discontinuation rates for pharmacotherapies are high. Given the gravity of the obesity epidemic in Mexico, successful weight management could have significant benefits to the patients and society at large.

\section{Acknowledgment}

This study was funded by Novo Nordisk.

\section{Disclosure}

At the time of this study, Marco D DiBonaventura and Allison Ehrenreich were employees of Kantar Health, which conducted the National Health and Wellness Survey. Marco D DiBonaventura and Allison Ehrenreich received funding to analyze and develop the manuscript from Novo Nordisk.
Henrik Meincke, Agathe Le Lay, Janine Fournier, and Erik Bakker are employees of Novo Nordisk. The authors report no other conflict of interest in this work.

\section{References}

1. Rtveladze K, Marsh T, Barquera S, et al. Obesity prevalence in Mexico: impact on health and economic burden. Public Health Nutr. 2014;17(1):233-239.

2. Soriano R, Ponce de León Rosales S, García R, García-García E, Méndez JP. High knowledge about obesity and its health risks, with the exception of cancer, among Mexican individuals. J Cancer Educ. 2012;27(2):306-311.

3. Cecchini M, Sassi F, Lauer JA, Lee YY, Guajardo-Barron V, Chisholm D. Tackling of unhealthy diets, physical inactivity, and obesity: health effects and cost-effectiveness. Lancet. 2010;376(9754):1775-1784.

4. Colchero MA, Sosa-Rubí SG. Heterogeneity of income and lifestyle determinants of body weight among adult women in Mexico, 2006. Soc Sci Med. 2012;75(1):120-128.

5. Jiménez-Cruz A, Manuel Loustaunau-López V, Bacardi-Gascón M. The use of low glycemic and high satiety index food dishes in Mexico: a low cost approach to prevent and control obesity and diabetes. Nutr Hosp. 2006;21(3):353-356.

6. Barquera S, Campos-Nonato I, Aguilar-Salinas C, Lopez-Ridaura $\mathrm{R}$, Arredondo A, Rivera-Dommarco J. Diabetes in Mexico: cost and management of diabetes and its complications and challenges for health policy. Global Health. 2013;9:3

7. Gupta S, Richard L, Forsythe A. The humanistic and economic burden associated with increasing body mass index in the EU5. Diabetes Metab Syndr Obes. 2015;8:327-338.

8. Valencia-Flores M, Orea A, Castaño VA, et al. Prevalence of sleep apnea and electrocardiographic disturbances in morbidly obese patients. Obes Res. 2000;8(3):262-269.

9. Castro-Rios A, Nevárez-Sida A, Tiro-Sánchez MT, Wacher-Rodarte N. Triggering factors of primary care costs in the years following type 2 diabetes diagnosis in Mexico. Arch Med Res. 2014;45(5):400-408.

10. López-Hernández D. Epidemiological association between body fat percentage and cervical cancer: a cross-sectional population-based survey from Mexico. Arch Med Res. 2013;44(6):454-458.

11. Rodríguez-Flores M, Rodríguez-Saldaña J, Cantú-Brito C, AguirreGarcía J, Alejandro GG. Prevalence and severity of atherosclerosis in different arterial territories and its relation with obesity. Cardiovasc Pathol. 2013;22(5):332-338.

12. Hitt HC, McMillen RC, Thornton-Neaves T, Koch K, Cosby AG. Comorbidity of obesity and pain in a general population: results from the Southern Pain Prevalence Study. J Pain. 2007;8(5):430-436.

13. Ray L, Lipton RB, Zimmerman ME, Katz MJ, Derby CA. Mechanisms of association between obesity and chronic pain in the elderly. Pain. 2011;152(1):53-59.

14. Urquhart DM, Berry P, Wluka AE, et al. 2011 Young Investigator Award winner: increased fat mass is associated with high levels of low back pain intensity and disability. Spine (Phila Pa 1976). 2011;36(16):1320-1325

15. Wright LJ, Schur E, Noonan C, Ahumada S, Buchwald D, Afari N. Chronic pain, overweight, and obesity: findings from a communitybased twin registry. J Pain. 2010;11(7):628-635.

16. Lementowski PW, Zelicof SB. Obesity and osteoarthritis. Am J Orthop (Belle Mead NJ). 2008;37(3):148-151.

17. Grotle M, Hagen KB, Natvig B, Dahl FA, Kvien TK. Obesity and osteoarthritis in knee, hip and/or hand: an epidemiological study in the general population with 10 years follow-up. BMC Musculoskelet Disord. 2008;9:132.

18. Hart DJ, Spector TD. The relationship of obesity, fat distribution and osteoarthritis in women in the general population: the Chingford Study. J Rheumatol. 1993;20(2):331-335.

19. Resta O, Foschino Barbaro MP, Bonfitto P, et al. Low sleep quality and daytime sleepiness in obese patients without obstructive sleep apnoea syndrome. J Intern Med. 2003;253(5):536-543. 
20. Resnick HE, Carter EA, Aloia M, Phillips B. Cross-sectional relationship of reported fatigue to obesity, diet, and physical activity: results from the third national health and nutrition examination survey. J Clin Sleep Med. 2006;2(2):163-169.

21. Akinnusi ME, Saliba R, Porhomayon J, El-Solh AA. Sleep disorders in morbid obesity. Eur J Intern Med. 2012;23(3):219-226.

22. Vgontzas AN, Kales A. Sleep and its disorders. Annu Rev Med. 1999;50: $387-400$.

23. Vgontzas AN, Tan TL, Bixler EO, Martin LF, Shubert D, Kales A. Sleep apnea and sleep disruption in obese patients. Arch Intern Med. 1994;154(15):1705-1711.

24. Luppino FS, de Wit LM, Bouvy PF, et al. Overweight, obesity, and depression: a systematic review and meta-analysis of longitudinal studies. Arch Gen Psychiatry. 2010;67(3):220-229.

25. de Hollander EL, Picavet HS, Milder IE, Verschuren WM, Bemelmans WJ, de Groot LC. The impact of long-term body mass index patterns on health-related quality of life: the Doetinchem Cohort Study. Am J Epidemiol. 2013;178(5):804-812.

26. Dee A, Kearns K, O'Neill C, et al. The direct and indirect costs of both overweight and obesity: a systematic review. BMCRes Notes. 2014;7:242.

27. DiBonaventura M, Lay AL, Kumar M, Hammer M, Wolden ML. The association between body mass index and health and economic outcomes in the United States. J Occup Environ Med. 2015;57(10):1047-1054.

28. Gates DM, Succop P, Brehm BJ, Gillespie GL, Sommers BD. Obesity and presenteeism: the impact of body mass index on workplace productivity. J Occup Environ Med. 2008;50(1):39-45.

29. Sanchez Bustillos A, Vargas KG 3rd, Gomero-Cuadra R. Work productivity among adults with varied Body Mass Index: results from a Canadian population-based survey. J Epidemiol Glob Health. 2015;5(2):191-199.

30. Barquera S, Campos I, Rivera JA. Mexico attempts to tackle obesity: the process, results, push backs and future challenges. Obes Rev. 2013;14 Suppl 2:69-78.

31. Elizondo-Montemayor L, Gutierrez NG, Moreno DM, Martínez U, Tamargo D, Treviño M. School-based individualised lifestyle intervention decreases obesity and the metabolic syndrome in Mexican children. J Hum Nutr Diet. 2013;26 Suppl 1:82-89.

32. Kushner RF. Weight loss strategies for treatment of obesity. Prog Cardiovasc Dis. 2014;56(4):465-472.

33. McLean N, Griffin S, Toney K, Hardeman W. Family involvement in weight control, weight maintenance and weight-loss interventions: a systematic review of randomised trials. Int J Obes Relat Metab Disord. 2003;27(9):987-1005.

34. Sarwer DB, von Sydow Green A, Vetter ML, Wadden TA. Behavior therapy for obesity: where are we now? Curr Opin Endocrinol Diabetes Obes. 2009;16(5):347-352.

35. Méndez-Hernández P, Dosamantes-Carrasco D, Lamure M, et al. Weight-loss practices among university students in Mexico. Int J Public Health. 2010;55(3):221-225.

36. United States Census Bureau. International data base. 2015. Available from: http://www.census.gov/population/international/data/idb/informationGateway.php. Accessed July 10, 2016.
37. Liu H, Cella D, Gershon R, et al. Representativeness of the patientreported outcomes measurement information system Internet panel. $J$ Clin Epidemiol. 2010;63(11):1169-1178.

38. DiBonaventura M, Richard L, Kumar M, Forsythe A, Flores NM, Moline $\mathrm{M}$. The association between insomnia and insomnia treatment side effects on health status, work productivity, and healthcare resource use. PLoS One. 2015;10(10):e0137117.

39. Dibonaventura MD, Yuan Y, Lescrauwaet B, et al. Multicountry burden of chronic hepatitis $\mathrm{C}$ viral infection among those aware of their diagnosis: a patient survey. PLoS One. 2014;9(1):e86070.

40. Gupta S, Isherwood G, Jones K, Van Impe K. Productivity loss and resource utilization, and associated indirect and direct costs in individuals providing care for adults with schizophrenia in the EU5. Clinicoecon Outcomes Res. 2015;7:593-602.

41. Laks J, Goren A, Dueñas H, Novick D, Kahle-Wrobleski K. Caregiving for patients with Alzheimer's disease or dementia and its association with psychiatric and clinical comorbidities and other health outcomes in Brazil. Int J Geriatr Psychiatry. 2016;31(2):176-185.

42. Vietri J, Otsubo T, Montgomery W, Tsuji T, Harada E. Association between pain severity, depression severity, and use of health care services in Japan: results of a nationwide survey. Neuropsychiatr Dis Treat. 2015;11:675-683.

43. Charlson ME, Charlson RE, Peterson JC, Marinopoulos SS, Briggs WM, Hollenberg JP. The Charlson comorbidity index is adapted to predict costs of chronic disease in primary care patients. JClin Epidemiol. 2008;61(12): 1234-1240.

44. Bang H, Edwards AM, Bomback AS, et al. Development and validation of a patient self-assessment score for diabetes risk. Ann Intern Med. 2009;151(11):775-783.

45. Rabin R, de Charro F. EQ-5D: a measure of health status from the EuroQol Group. Ann Med. 2001;33(5):337-343.

46. Reilly MC, Zbrozek AS, Dukes EM. The validity and reproducibility of a work productivity and activity impairment instrument. Pharmacoeconomics. 1993;4(5):353-365.

47. Organisation for Economic Co-operation and Development (OECD) [webpage on the Internet]. OECD Economic Surveys: Mexico. 2015. Paris: OECD Publication; 2015. Available from: http://dx.doi. org/10.1787/eco_surveys-mex-2015-en. Accessed July 10, 2016.

48. Lofland JH, Pizzi L, Frick KD. A review of health-related workplace productivity loss instruments. Pharmacoeconomics. 2004;22(3): 165-184.

49. Sullivan PW, Ghushchyan V. Preference-based EQ-5D index scores for chronic conditions in the United States. Med Decis Making. 2006;26(4):410-420.

50. Gupta S, Wang Z, Powers A. Patient survey on weight loss medication use in the US. Poster presented at: Obesity Week; November 2-7, 2014; Boston, MA.

51. Ogden CL, Carroll MD, Kit BK, Flegal KM. Prevalence of childhood and adult obesity in the United States, 2011-2012. JAMA. 2014;311(8): 806-814.

Diabetes, Metabolic Syndrome and Obesity: Targets and Therapy

Dovepress

\section{Publish your work in this journal}

Diabetes, Metabolic Syndrome and Obesity: Targets and Therapy is an international, peer-reviewed open-access journal committed to the rapid publication of the latest laboratory and clinical findings in the fields of diabetes, metabolic syndrome and obesity research. Original research, review, case reports, hypothesis formation, expert opinion and commentaries are all considered for publication. The manuscript management system is completely online and includes a very quick and fair peer-review system, which is all easy to use. Visit http://www.dovepress.com/testimonials.php to read real quotes from published authors. 